\title{
Recent Progress in Antitumoral Nanotechnology
}

\section{Felipe Barrera Méndez ${ }^{1,2}$, Francisco Javier Enríquez Medrano ${ }^{3}$, Nicolaza Pariona', Ramón Díaz de León Gómez ${ }^{3}$ and José Luis Olivares Romero ${ }^{1 *}$}

\author{
${ }^{1} R e d$ de Estudios Moleculares Avanzados, Clúster Científico y Tecnológico Biomimic $®$, Instituto de Ecología. \\ Carretera antigua a Coatepec 351, El Haya, Xalapa 91070, Veracruz, México \\ ${ }^{2}$ Catedratico CONACYT \\ ${ }^{3}$ Centro de Investigación en Química Aplicada, Blvd. Enrique Reyna, No. 140, 25294, Saltillo, Coahuila, México
}

\begin{abstract}
The last advances in applications of nanotechnology in antitumoral prevention, detection and treatment during the last decade are reviewed. Drug delivery and negative side effects are the seminal challenges in order to get more effective cancer therapies. In this context, nanotechnology provides a quite versatile platform to deal with it. Herein is discussed the recent state-of-the-art of nanotechnology, which includes: metal nanoparticles, quantum dots, micelles, nanoencapsulation, polymeric nanoparticles, liposomes, ceramic-based carriers, dendrimers, nanoshells, and carbon nanotubes. Finally, the review discusses the advantages and challenges for the application of nanotechnology in antitumoral therapy.
\end{abstract}

\section{Keywords}

Nanotechnology, Nanoparticles, Antitumoral, Cancer therapy.

\section{Introduction}

Cancer arises when a group of cells begin to grow abnormally disregarding the division rules of normal cells. Because of their uncontrolled growth and proliferation, these cancer cells establish an autonomous tumor tissue. Metastasis, the spreading of malignant tumor cells, is the main cause of cancer lethality. Cancer is a leading cause of morbidity and mortality worldwide. In cancer therapy, undoubtedly the first and the most important step is diagnosis [1]. It is obvious that the earlier the cancer diagnosis, the better the chance for successful treatment and survival. Thus, the detection time frame has an enormous effect on a patient's prognosis, nanotechnology bring new hope to the arena of cancer detection research, owing to nanoparticles' unique physical and chemical properties, giving them the potential to be used as a synthetic scaffold for imaging probes in the detection and monitoring of cancer. Nanoparticle (NPs) is defined as an aggregate of atoms bonded together with a radius between 1 and $100 \mathrm{~nm}$ [2]. Materials of this size are especially interesting because of their physical and chemical properties (e.g., lower melting points, higher specific surface areas, specific optical properties, mechanical strengths, and specific magnetizations) differs significantly from those observed in bulk materials and this properties that might prove attractive in various field applications [3]. NPs can be based on polymers, metals, oxides, etc., and according to the characteristic desired, not only depends of precursor salts, but also it will depend of the synthesis method. As a result, strict control of the nanoparticles morphology is therefore required in order to obtain NPs of desired properties. The synthesis of NPs can be by top-down, which is the breakdown method; an external force is applied to a solid that leads to its break-up into smaller particles. The other way to produce NPs and the most used is the bottom-up; which is departed from atoms of gas or liquids based on atomic transformations or molecular condensations $[3,4]$ and includes chemical and physical routes. One of the interesting field applications can be found in nanomedicine, where NPs can improve diagnosis, treatment, drug delivery system, bio tagging or labeling, etc [5-8]. Particularly, NPs have received great attention in antitumoral treatment and currently research have shown that different metal and metal oxide NPs are effective in cancer therapy and diagnosis [9]. This NPs have antitumor activity by themselves or in combination with other therapies [10]. The most commonly studied NPs include Gold NPs [5], iron oxide NPs [11], quantum dots [12], polymeric NPs [13], carbon nanotubes [14] and liposomes [15].

\section{Gold Nanoparticles}

Gold nanoparticles AuNPs have unique physical, chemical, optical

*Corresponding author: José Luis Olivares Romero, Red de Estudios Moleculares Avanzados, Clúster Científico y Tecnológico Biomimic $®$, Instituto de Ecología. Carretera antigua a Coatepec 351, El Haya, Xalapa 91070, Veracruz, México,E-mail: jose.olivares@inecol.mx

Received: October 16, 2015: Accepted: November 28, 2015: Published: December 02, 2015

Copyright: (c) 2015 Méndez FB. This is an open-access article distributed under the terms of the Creative Commons Attribution License, which permits unrestricted use, distribution, and reproduction in any medium, provided the original author and source are credited. 
and electronic properties, which can be used in multidisciplinary research. There are different methods to synthesize AuNPs, and the chemical synthesis is the easiest and less toxicway. Among the conventional methods to obtain AuNPs, the citrate reduction is the most popular, because this method is simple, cheap, and can be used to prepare large quantities of NPs [16]. AuNPs have especially been attractive due to optical properties, which are conferred when sized AuNPs are excited with a wavelength much smaller than that of the of incident light, then an electric field is formed in the particle, which at a certain wavelength induce a resonance of the free electrons across the particle, this is known as surface plasmon resonance (SPR), then the particle absorbs and scatters the electromagnetic radiation intensely [17]. The resonance frequencies are strongly dependent on the gold nanoparticle size, surface and agglomerations [18,19]. The plasmon resonance properties of AuNPs have been explored for applications in nanomedicine with emphasis on cancer, which can enhanced biological labeling, detections, targeted therapy, diagnostic and sensing [20-23]. Also, AuNPs can be used as drugdelivery carries due to biocompatibility and immunogenicity [24] For instance, Figueroa et al.[25], have developed a gold nanoparticle (AuNP) polyamidoamine (PAMAM) conjugates for use as non-viral transfection agents. AuPAMAM conjugates were prepared by cross linking PAMAM dendrimers to carboxylic-terminated AuNPs via EDC and sulfo-NHS chemistry. The results showed that increasing the amine to carboxyl ratio during conjugation of PAMAM onto AuNPs yielded the optimal vector with respect to colloidal stability and transfection efficiency in vitro. AuPAMAM conjugates present attractive candidates for non-viral gene delivery due to their commercial availability, ease of fabrication and scaleup, high yield, high transfection efficiency and low cytotoxicity. Additionally, a study about biocompatibility and cancer therapeutic applications using glycoprotein (GA) functionalized AuNPs containing a $b$-emitting Au-198 were used in compromised immunodeficient (SCID) mice that present human prostate tumor xenografts. The results showed significant tumor regression and effective control in the growth of prostate tumors over 30 days. Three weeks later after administration of GA-198AuNPs, tumor volumes for the treated animals were $82 \%$ smaller when compared with tumor volume of control group. The results provide further evidence on the therapeutic efficacy and concomitant in vivo tolerance and nontoxic features of GA-198AuNPs [26]. Moreover, the study of AuNPs as radiation sensitizer in cancer therapy shows that the highest radio sensitization enhancement factor was AuNPs of $50 \mathrm{~nm}(1.43$ at $220 \mathrm{k} \mathrm{Vp})$ compared to AuNPs of 14 and $74 \mathrm{~nm}$ (1.20 and 1.26, respectively) [22]. Plasmonic photothermal therapy (PPTT) and Photo-thermal therapy (PTT) are a minimalinvasive therapy in which photon energy is converted into heat to kill cancer. Due to its plasmonic properties, AuNPs have been widely used to enhance this treatment thereby making them superior contrast agents for PTT $[27,28]$. In this sense, Mackey et al. [29], utilized theoretical calculations as well as experimental techniques in vitro to determine the optimum gold nanorod (AuNR) size, it was compared the plasmonic properties and the efficacy as photothermal contrast agents of three different sizes of AuNRs. Examining three different AuNRs $(38 \times 11,28 \times 8$, and $17 \times 5 \mathrm{~nm})$, was determined that the 28 $\times 8 \mathrm{~nm}$ AuNR is the most effective in plasmonic photothermal heat generation. The $28 \times 8 \mathrm{~nm}$ AuNR was found to be the most effective photothermal contrast agent for PPTT in human oral squamous cell carcinoma.

\section{Magnetic Nanoparticles}

Iron and its compounds are widespread in nature and can be readily synthesized in the laboratory [30]. Nowadays, the synthesis and utilization of iron oxide nanoparticles with unique properties has been widely studied because of their potential applications in many field such as physics, chemistry, environmental, biology and medicine, particularly, due to magnetic properties and biocompatibility such as magnetite $\left(\mathrm{Fe}_{3} \mathrm{O}_{4}\right)$ and maghemite $\left(\gamma-\mathrm{Fe}_{2} \mathrm{O}_{3}\right)$. Magnetic properties are principally sensitive to the particles size, when size of particle decreases; it can exhibit superparamagnetic phenomena due to each particlecan be considered as a single magnetic domain [31]. For that reason, the synthetic route to get NPs is highly important, many different synthetic procedures have been developed, but based in bottom-up synthetic procedures, the most common are: co-precipitation, microemulsion, and sonochemical synthesis [32]. Super paramagnetic nanoparticles (SPMN) are biocompatible and offer a high potential for several biomedical applications. Therefore, SPMN have been attracted much attention in a great variety of applications in biological and medicine, such as biosensing, drug delivery, hyperthermia, cell labeling and as contrast agents [33-35]. The foregoing, based on their inducible magnetization, this magnetic NPs can be directed to a defined location and heated in presence of an external magnetic field [35].

Recently, superparamagnetic nanoparticles have attracted a lot of attention in diagnosis and therapies for cancer treatment. Some studies have showed that the use of SPMN to diagnose tumor malignancy accurately and in early states would minimize the cost in clinical practices. A study of fabrication and characterization of thermally cross-linked superparamagnetic ironoxide nanoparticles (TCL-SPION) was developed in order to detect tumors in vivo. When the Cy5.5 TCL-SPION was administered to mice with Lewis lung carcinoma tumor by intravenous injection, the tumor was unambiguously detected in $\mathrm{T} 2$-weighted magnetic resonance images as a $68 \%$ signal drop as well as in optical fluorescence images within $4 \mathrm{~h}$, these result indicate a high level of accumulation of the nanomagnets within the tumor site [36].

SPMN can be used alone or with surface modification. SPMN could play an important role in the development of hyperthermia for treatment of tumors in vivo. Size and surface modification of MNPenable a safe application to the tumor and high heating efficiency by Neel relaxation, which promises good therapeutic success [37]. It is important to highlight that SPMN with functionalized surface coatings can conjugate chemotherapeutic drugs or be used to target ligands/proteins, making them usefulfor drug delivery, targeted therapy, magnetic resonance imaging, transfection, and cell/protein/ DNA separation $[38,39]$.

Recently some studies have demonstrated that catalytic activity of SPMN can improve the anticancer drug efficacy. This is because SPMN in presence of hydrogenperoxide generate reactive oxygen species (ROS) [40,41]. In fact, Huang et al. [11], have reported that $\mathrm{pH}$-responsive SPION-micelles can synergize with $\beta$-lap to improve cancer therapy. These SPION-micelles, selectively release iron ions inside cancer cells, which interact with hydrogen peroxide generated from $\beta$-lap in a tumor-specific, in an NQO1-dependent manner. Through Fenton reactions, these iron ions escalate the ROS stress in $\beta$-lap-exposed cancer cells, there by greatly enhancing the therapeutic index of $\beta$-lap. It was suggested that the incorporation of SPIONmicelles with ROS-generating drugs could potentially improve drug efficacy during cancer treatment [11].

\section{Quantum Dots}

Quantum dots (QDs) are semiconductor nanocrystals, which due to their unique photophysical, mechanical, electrochemical, and catalytic properties have attracted great attention for applications, particularly in electronics, optoelectronic, biology and medicine $[42,43]$. QDs properties are size-dependent due to the quantum confinement effect [44]. The absorption onset and florescence emission shift to larger energy while decreasing size [45]. In other words, QDs could vary in color from green-yellow to orange-red and luminesce from blue to yellow, where shorter wavelength, higher energy, electronic transitions correspond to smaller crustal size [46]. It is important to control in a relatively simple manner the variation of 
core size and compositions, and through variation of surface coating [44], which will depends of the QDs synthesis. The most common way to obtain QDs is by bottom-up processes [42]. Particularly, wet chemical method, which mainly follow conventional precipitation method with careful control of parameters for a single solution or mixture of solution [42].

For medical applications QDs could provide a versatile nanoscale scaffold, whereby it can design multifunctional QDs with both imaging and therapeutic functions. The narrow emission and broad absorption spectra of QDs makes them well suited to excellent multiplexed bioimaging, cell tracking, and diagnostic applications [47,48], specially for cancer treatment. Nowadays, due to QDs advantages, there are several studies related with applications in cancer treatment. The folate receptor is over expressed in a broad spectrum of malignant tumors that makes it an attractive target for selective delivery of imaging agents to tumor cells [49]. Nanoparticles containing QDs entrapped in a lipid shell, and then post-loaded with a folate-lipid conjugate for targeting in mouse and human tumor cells expressing the folate receptor were studied. As result it was found that only folate-targeted lipodots were taken up by tumor cells. Confocal depth scanning showed substantial internalization, which confirms the specificity of targeted folate lipodots. Besides, binding and internalization were inhibited by free folate, and no uptake was found in a folate-receptor negative cell line [49].

The biocompatible luminescent properties of QDs, have allowed to develop a highly stable aqueous suspensions of Silicon QDs using phospholipid micelles, in which the optical properties of $\mathrm{Si}$ nanocrystals are retained. These luminescent micelle-encapsulated Si QDs were used as luminescent labels for pancreatic cancer cells [50]. Another applications of QDs are as chemosensors Lemon et al. [43], designed optical chemosensors that feature a quantum dot and an analyte-responsive dye. It was reported that chemosensors is noninvasive, and allows the dynamical monitoring of $\mathrm{pH}$, oxygen, and glucose, within the tumor microenvironment by using multiphoton imaging.

\section{Polymeric Nanoparticles}

It is possible to overcome the limitations of the nanotechnology's applications in medicine, such as toxicity and instability, by anchoring biocompatible polymers on the surfaces of diagnostic nanomedicines [51,52]. The surface modification of nanoparticles with hydrophilic polymers reduces the interfacial energy in an aqueous environment, thus preventing unwanted aggregation due to secondary interactions between nanoparticles. In addition, changing the surface of nanoparticles with hydrophilic polymers would minimize the recognition by proteins and cells in the body, which allows to nanomedicine circulate in the blood stream for a longer period of time, hence increasing the odds to reach the target site.

Polymers also bring an option to develop drug delivery systems, used as therapeutics in cancer treatments, holding the anticancer drug in the blood and then allowing a burst or continuous drug release at the cancer tissue, consequently reducing the dosage of the medicine and the collateral toxicity of the treatment. The drug is typically either dispersed within the polymeric nanoparticle, where the encapsulated drugs are gradually released from the polymer matrix by diffusion, or conjugated to the polymeric backbone, where degradation of the polymer matrix can play a primary role in drug release, and various techniques may be used to adjust the release rate.

In addition to simple conjugates based on linear or branched polymers, intricate polymeric conjugates have recently been produced using multivalent polymers, graft polymers, dendrimers, dendronized polymers, block copolymers and star-shaped polymers [53]. Conjugated polymers have also been used to form polymeric micelles for delivery of poorly soluble drugs [54-56].

\section{Polymer Nanoencapsulation}

Nanoencapsulation of drugs involves forming drug-loaded particles with diameters ranging from 1 to $1000 \mathrm{~nm}$. Nanocapsules are vesicular systems in which the drug is confined to a core surrounded by a polymeric membrane [57]. The submicron size of nanoparticles offers a number of distinct advantages over microparticles, including a higher intracellular uptake [58].

The nanoencapsulation can be achieved either using monomers to perform a polymerization reaction, or may be achieved directly from a macromolecule or preformed polymer [57].

\section{Emulsion polymerization}

Emulsion polymerization is one of the fastest methods for nanoparticle preparation and is readily scalable [59]. The method is classified into two categories, based on the use of an organic or aqueous continuous phase. The continuous organic phase methodology involves the dispersion of monomer into an emulsion or inverse microemulsion, or into a material in which the monomer is not soluble (non solvent). As one of the first methods for production of nanoparticles, surfactants or protective soluble polymers were used to prevent aggregation in the early stages of polymerization [60]. This procedure requires toxic organic solvents, surfactants, monomers and initiators, which are subsequently eliminated from the formed particles. In the aqueous continuous phase, the monomer is dissolved in a continuous phase that is usually an aqueous solution, and the surfactants or emulsifiers are not needed. The polymerization process can be initiated when a monomer molecule dissolved in the continuous phase collides with an initiator molecule that might be an ion or a free radical. Alternatively, the monomer molecule can be transformed into an initiating radical by high-energy radiation, including $\gamma$-radiation, or ultraviolet or strong visible light. Chain growth starts when initiated monomer ions or monomer radicals collide with other monomer molecules according to an anionic polymerization mechanism [61].

\section{Emulsification/solvent evaporation}

An organic phase containing the dissolved drug and polymer is dispersed into nanodroplets, using a dispersing agent and highenergy homogenization [62] in a non solvent or suspension medium such as chloroform or ethyl acetate. The polymer precipitates in the form of nanospheres in which the drug is finely dispersed in the polymer matrix network. The solvent is subsequently evaporated by increasing the temperature under pressure or by continuous stirring. This method can only be applied to lipid soluble drugs, and limitations are imposed by the scale-up of the high-energy requirements in homogenization [63].

\section{Solvent displacement and interfacial deposition}

The polymer is dissolved in a water-miscible solvent of intermediate polarity, leading to the precipitation of nanospheres. This phase is injected into a stirred aqueous solution containing a stabilizer as a surfactant. The fast diffusion of the solvent provokes deposition of the polymer on the interface between the water and the organic solvent, leading to the instantaneous formation of a colloidal suspension [64]. The usefulness of this simple technique is limited to water-miscible solvents, where the diffusion rate is enough to produce spontaneous emulsification. This method, as well as the emulsification/solvent evaporation method, is applicable to lipophilic drugs [65].

\section{Emulsification/solvent diffusion}

In this method the encapsulated polymer is dissolved in a partially water soluble solvent, such as propylene carbonate, and saturated with water to ensure the initial thermodynamic equilibrium of both liquids. Subsequently, the polymer-water saturated solvent phase is 
emulsified in an aqueous solution containing the stabilizer, leading to solvent diffusion to the external phase and the formation of either nanospheres or nanocapsules, according to the oil-to-polymer ratio. Finally, the solvent is eliminated by evaporation or filtration. As well as the other techniques that use a preformed polymer for encapsulation, this method is efficient encapsulating lipophilic drugs [64].

In this context, Jie et al. synthesized amphiphilic $N$-(2-hydroxy)propyl-3-trimethylammonium-chitosan-cholic acid polymers by unification of cholic acid and glycidyltrimethylammonium chloride onto chitosan and self-assembled into nanoparticles in phosphate-buffered saline. Doxorubicin could be encapsulated into these nanoparticles and then could easily be up taken by breast cancer (MCF-7) cells and released into the cytoplasm [66]. Another interesting example was developed by Shengtang et al., synthesize folic acid-conjugated chitosan-polylactide copolymers to build a drug carrier with active targeting of paclitaxel. Targeting characteristic was confirmed using folic acid receptor-expressed MCF-7 breast cancer cells [67]. Also, Park et al., reported that glycol chitosanbased nanoparticles of adriamycin are useful for sustained and specific delivery of adriamycin, thus showing lower cytotoxicity than adriamycin alone [68].

\section{Liposomes}

Liposomes are spherical vesicles that have at least one lipid bilayer, as opposed to the micelles, which are composed of monolayers. A liposome encapsulates an aqueous solution inside a hydrophobic membrane and dissolved hydrophilic solutes cannot readily pass through the lipids. Hydrophobic chemicals can be dissolved into the membrane, and in this way liposome can carry both hydrophobic molecules and hydrophilic molecules. The diameter of these spheres goes from $50 \mathrm{~nm}$ to $1000 \mathrm{~nm}$, making them a convenient delivery vehicle for biologically active compounds [69]. Liposomes are manufactured in majority using various procedures in which the water soluble (hydrophilic) materials are entrapped by using aqueous solution of these materials as hydrating fluid or by the addition of drug/drug solution at some stage during manufacturing of the liposomes. The lipid soluble (lipophilic) materials are solubilized in the organic solution of the constitutive lipid and then evaporated to a dry drug containing lipid film followed by its hydration. These methods involve the loading of the entrapped agents before or during the manufacturing procedure (passive loading). Certain type of compounds with ionizable groups, as well as those, which display both lipid and water solubility, can be introduced into the liposomes after the formation of intact vesicles (remote loading). Liposomes have the advantages of biological degradability and relative toxicological and immunological safety, and have diagnostic and therapeutic applications. For diagnosis, aqueous contrastenhancing agents entrapped in liposomal carriers can be targeted to the liver and spleen, and distinctions can be made between normal and tumorous tissue using computed tomography [70,71], and gas filled liposomes are used for diagnostic ultrasound and magnetic resonance imaging due to their differing magnetic susceptibility and ability to reflect sound well [72,73]. Therapeutics liposomes have been used to deliver anticancer agents in order to reduce the toxic effects of the drugs when given alone or to increase the circulation time and effectiveness of the drugs as show by Uziely et al., they utilized a formulation of doxorubicin encapsulated in polyethylene glycol coated which improved the pharmacokinetics of free doxorubicin [74]. Northfelt et al., treated fifty-three patients with advanced Kaposi's sarcoma refractory to standard therapy, with Doxil ${ }^{\circ}$. One patient $(2 \%)$ had a complete response whereas a partial response was observed in 19 patients (36\%) [75]. Stage IV breast cancer patients also benefited from the treatment with liposomal doxorubicin according to a study by Ranson et al. complete and partial responses were obtained in $6 \%$ and $25 \%$ respectively [76]. Muggia et al., found that liposomal doxorubicin had substantial activity against refractory ovarian cancer [77]. Wolf et al. [58], have studied the delivery of a liposome-encapsulated DNA repair enzyme, T4 endonuclease $\mathrm{V}$, in $\mathrm{pH}$-sensitive liposomes composed of phosphatidylcholine, phosphatidylethanolamine, oleic acid and cholesteryl hemisuccinate (2:2:1:5 molar ratio). Liposome encapsulation enhanced the delivery of T4 endonuclease across mouse skin in vivo leading to increased protection against UV radiation. This reduced the incidence of skin cancer in a UV-induced mouse model and prevented systemic immunosuppression of contact and delayed type of hypersensitivity.

\section{Micelles}

A micelle is an aggregate of molecules having both polar and non-polar regions (amphiphilic molecules) dispersed in an aqueous solution. In a typical micelle the polar or hydrophilic heads form an outer shell in contact with water, while non polar or hydrophobic tails are sequestered in the interior. Micelles are widely used in industrial and biological fields for their ability to dissolve and move non polar substances through an aqueous medium, or to carry drugs which are, often, scarcely soluble in water.

In an aqueous solution, micelles with core-shell structures are formed through the segregation of insoluble hydrophobic blocks into the core, which is surrounded by a shell composed of hydrophilic blocks. In general, there are three major methods for loading drugs into polymer micelle cores: chemical conjugation, physical entrapment or solubilization, and finally, polyionic complexation.

\section{Chemical conjugation}

For this method a drug is chemically conjugated to the coreforming block of the copolymer via a carefully designed $\mathrm{pH}$ - or enzyme-sensitive linker that can be cleaved to release a drug in its active form within a cell. The polymer-drug conjugate then acts as a polymer prodrug, which self assembles into a core-shell structure [53,78]. Bae et al. [79], prepared targeted anti-cancer micelles by conjugating folate to the distal end of poly (ethilen glycol)- $b$ poly(aspartate) block copolymer, or PEG-PAsp, aimed at increasing tumor accumulation. The folate-conjugated polymeric micelle loaded with Dox showed significantly increased cellular uptake. Cytotoxicity analysis in vitro indicated that the cell growth-inhibitory activity of the folate-conjugated micelle was enhanced, suggesting that this could be an effective approach for ligand-mediated uptake for cancer treatment [79].

\section{Physical entrapment}

This method is preferred for hydrophobic drug molecules. A variety of drugs can be physically incorporated into the core of the micelles by engineering the structure of the core-forming segment. Different loading methods can be used for physical entrapment of the drug into the micelles, such as dialysis [80], oil in water emulsification [81], direct dissolution [82], or solvent evaporation techniques [83].

Tumor-specific targeting of polymer micelles to molecular markers expressed at the surface of the cancer cells has been also explored to eradicate tumor cells. Mixed micelles of poly (ethylene oxide) (PEO)- $b$-poly (L-histidine) and PEO- $b$-poly(L-lactic acid) block copolymers with solubilized doxorubicin or micelles of PEO$b$-poly(DL-lactic-co-glycolic acid) block copolymer with covalently attached doxorubicin each were surface modified by conjugating folate molecules to the free PEO ends $[84,85]$. In both cases in vitro and in vivo studies demonstrated increased antitumor activity of the micelle incorporated drug resulting from such modification.

\section{Polyionic complexation}

Charged therapeutic agents can be incorporated into block copolymer micelles through electrostatic interactions with an oppositely charged ionic segment of block copolymer. This approach is now widely used for the incorporation of various polynucleic acids 
into block ionomer complexes for developing non-viral gene delivery systems.

As an example, the metal-complex formation of ionic block copolymer, PEO-b-poly(L-aspartic acid), was explored by Yokoyama et al. to prepare polymer micelles incorporating cis-dichlorodiamminoplatinum (II) (CDDP) [86], a potent chemotherapeutic agent widely used in the treatment of a variety of solid tumors, particularly, testicular, ovarian, head and neck, and lung tumors $[87,88]$. The CDDP-loaded micelles had a size of approximately $20 \mathrm{~nm}$. These micelles showed remarkable stability upon dilution in distilled water, while in physiological saline they displayed sustained release of the regenerated Pt complex over 50 $\mathrm{h}$ due to inverse ligand exchange from carboxylate to chloride. The release rate was inversely correlated with the chain length of poly (L aspartic acid) segments in the block copolymer. The stability of CDDP loaded micelle against salt was shown to be improved by the addition of homopolymer, poly (L-aspartic acid), in the micelles. Recently, Nishiyama et al., prepared CDDP-loaded micelles using another block copolymer, PEO- $b$-poly(glutamic acid) to improve and optimize the micellar stability, as well as the drug release profile [89]. The drug loading in the micelles was as high as $39 \%(w / w)$, and these micelles released the platinum in physiological saline at $37^{\circ} \mathrm{C}$ in sustained manner for over $150 \mathrm{~h}$ without initial burst of the drug [90].

\section{Ceramic based carriers}

The use of nanoparticles as carriers is a very promising approach because these nanomaterials can be used as photosensitizing agents in cancer treatment as Photodynamic therapy (PDT). PDT involves the uptake of a photosensitizer by cancer tissue followed by photoirradiation. PDT has been used as a clinical treatment of cancer and particularly for the treatment of superficial tumors [91]. The properties of an ideal photosensitizer are: stable composition, easily synthesized or readily available, minimal self-aggregation tendency, not highly hydrophobic or encapsulated inside appropriate carries, non-toxic in the absence of light exposure, photostable, absorbance in the red region of spectrum with high extinction molar coefficient, target specifity, and has to be quickly cleared from the body. Recent advances have been made in the use of this nanoparticles in terms of stability, photocytotoxic efficiency, biodistribution and therapeutic efficiency. The possibility of using ceramic nanoparticles for Photodinamic therapy (PDT) was shown by Prasad et al. [92], it was encapsulated $\mathrm{HPPH}$, a photosensitizer that is currently undergoing phase I and II clinical trials for esophageal cancer [93], by controlled hydrolysis of triethylvinylsilane in micellar media. In vitro experiments showed significant levels of cell death for both HPPH-tween-80 micelles and HPPH-nanoparticles in which the photosensitizer were covalently attached to the silica matrix [94], and they were able to show that ${ }^{1} \mathrm{O}_{2}$ was deactivated mainly outside the nanoparticles [95].

\section{Dendrimers}

Fritz and coworkers first introduced the term dendrimer in 1978 [96]. The dendrimer architecture permits control over properties such as shape, size, polarity, reactivity, density and solubility. Dendrimers have stimulated wide interest in the field of chemistry and biology, especially in applications like drug delivery, gene therapy and chemotherapy. A treatment of cancer mainly focused on the targeting the active drug molecule at the site without affecting the neighbor cells and dendrimers have this property, which is useful in diagnosis and treatment purpose. For instance, Liposomal locked-in dendrimers (LLDs), the combination of liposomes and dendrimers in one formulation, represents a relatively new term in the drug carrier technology. Konstantinos et al. synthesized 1 and 2-hydroxyterminated dendrimers and were then locked in liposomes consisting of DOPC/DPPG. The anticancer drug doxorubicin (DOX) was loaded into pure liposomes or LLDs and the final products were subjected to lyophilization. The loading of DOX as well as its in vitro release rate from all systems was determined and the interaction of liposomes with dendrimers was assessed by thermal analysis and fluoresce spectroscopy. The results were very promising in terms of drug encapsulation and release rate, factors that can alter the therapeutic profile of a drug with low therapeutic index such as DOX. Physicochemical methods revealed a strong, generation dependent, interaction between liposomes and dendrimers that probably is the basis for the higher loading and lower drug release from the LLDs comparing to pure liposomes [97]. Hsueh-Chen et al. studied the changes in global gene-expression profiles in human cervical cancer HeLa cells exposed to non activates and activates poly(amidoamine) (PAMAM) dendrimers, alone or in complexes with plasmid DNA (dendriplexes). Real-time quantitative reverse transcriptase-polimerase chain reaction was used to confirm four regulated genes (PHF5A, ARNTL2, CHD4, and P2RX7) affected by activated dendrimers and dendriplexes alike induced multiple gene expression changes, some of which overlapped with their dendriplexes. Dendrimers and dendriplexes principally affect genes with the molecular functions of nucleic acid binding and transcription activity, metal-ion binding, enzyme activity, receptor activity, and protein binding. These findings provide a deeper insight into the changes in gene expression patterns caused by the molecular structure of PAMAM dendrimers for gene-based cancer therapy [98].

\section{Nanoshells}

Nanoshell-based complexes have recently been expanded to include two diagnostic capabilities, MRI and near-infrared fluorescence imaging [99], in addition to photothermal therapy which use the ability of nanoshells to convert absorbed light to heat $[100,101]$ For instance, Jianlin et al. used uniform Au NRs-capped magnetic core/mesoporus silica shell nanoellipsoids (Au NRsMMSNEs) prepared by coating a uniform layer of Au NRs on the outer surface of a magnetic core/mesoporous silica shell nanostructure. This multifunctional nanocomposite integrate simultaneous chemotherapy, photo-thermotherapy, in vivo MR-, IR thermal and optical imaging into one single system. The obtained multifunctional nanoellipsoids showed very low cytotoxicity, and the cancer cell up take and intracellular location of the nanoellipsoids were observed by confocal laser scanning microscopy and bio-TEM. It was shown the synergistic effect of combined chemo- and photo-thermotherapy [102]. Another interesting example was developed by Maoquan et al. gold $(\mathrm{Au})$ nanoshells with solid silica cores have great potential for cancer phtotothermal therapy. The silica shells were synthesized using nanoliposome templates, and the Au nanoshells were grown on the outer surface of the silica shells. After doxorubicin (DOX) was incorporated into liposome $/ \mathrm{SiO}_{2} / \mathrm{Au}$, the DOX-loaded Au nanoshells killed cancer with high therapeutic efficacy when irradiated with near-IR light, suggesting that the Au nanoshells delivered both DOX chemotherapy and photothermal therapy with a synergistic effect [102].

\section{Carbon nanotubes}

Carbon nanotubes as unique and novel class of nanomaterials have shown considerable promise in cancer therapy and diagnosis among the countless of nanocarriers. The presence of a large surface area enables the engineering of the surface of nanotubes, thus making them biocompatible, and large benefits can be harnessed from them. Together with their ability to encapsulate small molecules, stacking interactions and conjugation, nanotubes have improved the profile of anticancer agents. The propensity to absorb the body transparent NIR radiation also envisages photothermal and photoacoustic therapy using nanotubes $[1,103]$. For instance, Ojima et al. effectively employed carbon nanotubes-polymer hybrid nanomaterials to deliver biologically active therapeutic agents into the tumor site for the purposes of cancer diagnosis and therapy [104]. 
Li et al. use functionalized-CNTs in gene therapy because they are easily able to cross cell membranes and deliver genes into cells. In many reports, DNA and RNA have been covalently $[105,106]$ and noncovalently [107] attached to functionalized CNTs. It has been found that not only DNA molecules can be linked to the tips and walls of CNTs, but also can be encapsulated inside the structure [108]. Release of DNA molecules from CNTs-based delivery systems using thermal properties of CNTs has been investigated and it has been found that DNAs can be released from functionalized-CNTs transported to the nucleus after the laser pulses [109].

While scientist focus on the development of safer and more efficient techniques for rapid diagnosis and smart drug delivery in cancer therapy also the toxicity and immunogenicity of carbon nanotubes (CNTs) in both in vitro and in vivo studies has been studied and attributed to various factors such as number of walls, length and aspect ratio, surface area, degree of aggregation, extent of oxidation, hydrophobicity, surface topology, methods of administration, dispersibility, type and degree of functionalization, and method of manufacture. Although toxicity of CNTs is also dependent on their concentration, dose, duration and method that cells or organism are exposed to them and even the utilized dispersant to solubilize the nanotubes [1]

\section{Tumor environment and basis of tumor targeting mechanism}

There are two main ways in which nanoparticles can be delivered into the tumor tissue: by either passive or active targeting.

Passive targeting takes advantage of the inherent size of nanoparticles and the unique properties of tumor vasculature, such as the enhanced permeability and retention (EPR) effect and the tumor microenvironment [110-113].

In the EPR effect, the angiogenic blood vessels in tumor tissues (unlike those in normal tissues) have gaps as large as 600 to 800 $\mathrm{nm}$ between adjacent endothelial cells [114]. Defective vascular architecture coupled with poor lymphatic drainage induces the EPR effect [115], which allows nanoparticles to extravasate through these gaps into extravascular spaces and accumulate inside tumors tissues. The tumor drug accumulation can be achieved when a drug is delivered by a nanoparticle rather than as a free drug [116]. In general, the accumulation of nanoparticles in tumors depends on factors including the size, surface characteristic, and circulation halflife of the nanoparticle and the degree of angiogenesis of the tumor. Usually, less nanoparticle accumulation is seen in pre angiogenic or necrotic tumors.
Regarding to the Active targeting, the polymeric nanoparticles that have been tested clinically so far have mostly lacked a targeting moiety and instead rely mainly on the EPR effect of tumors, the tumor microenvironment, and tumor angiogenesis to promote some tumor selective delivery of nanoparticles to tumor tissues. An alternative strategy to overcome these limitations is to conjugate a targeting ligand or an antibody to nanoparticles. By incorporating a targeting molecule that specifically binds an antigen or receptor that is either uniquely expressed or overexpressed on the tumor cell surface, the ligand-targeted approach is expected to selectively deliver drugs to tumor tissues with greater efficiency. Indeed, several targeted polymeric nanoparticles are currently undergoing preclinical studies [117].

\section{Nano-formulated drugs}

As described previously drug delivery to solid tumors is one of the seminal challenges to developing more effective cancer therapies and it has exponentially increased in the last decades. Nanoparticles come in an almost infinite variety of sizes, shapes, and compositions, as a matter of fact it can be designed with multiple functionalities to aid in cancer therapies [118]. Nonetheless, the body possesses numerous defense mechanisms to protect itself against foreign substances including viruses, bacteria, protein toxins, and other chemicals [119]. Nanoparticles are no exception and are actively cleared by the body [120]. These defense mechanisms are in place at every level of organization, systemic, organ, tissue, cellular, and intracellular. On the other hand, most chemotherapeutic drugs are cytotoxic agents, which have specific targets for action inside a cancerous cell [121] and most of the time the drug administered to the patient becomes at best ineffective, and at worst toxic to the patient [122]. Nanotechnology might have a deep impact in solving many of the problems associated with conventional anti cancer drugs because nano-formulated drugs can be made as relatively safe, injectable formulations. Doxil and Abraxane are the two major nano-formulated drugs currently available on the market and already they have made an impact in cancer treatment worldwide. Doxil, which is doxorubicin formulated in nano-liposome [123], has shown significant improvements over its counterpart, free doxorubicin. Abraxane ${ }^{\infty}$ (Abraxis), with a size around $100 \mathrm{~nm}$, is an albumin-bound nanoparticle formulation of paclitalxel [124-126] and is widely used for treatment of metastatic breast cancer. The major advantage of Abraxane is that it evades the hypersensitivity reaction associated with Cremophor EL, the solvent used in conventional paclitaxel therapy. Thus, Abraxane clearly demonstrates the ability to convert insoluble or poorly soluble drugs, avoiding the need for toxic organic solvents. A list of the

Table 1: Nano-formulations commercial- and currently available.

\begin{tabular}{|c|c|c|c|c|c|}
\hline Product & Company & Drug & Formulation/ROA & Application & Status \\
\hline Abraxane & $\begin{array}{l}\text { Abrasix Bioscience, } \\
\text { AstraZeneca }\end{array}$ & Paclitaxcel & Albumin-bound nanoparticles-iv & Metastatic breast cancer & Marketed \\
\hline Caelyx & Schering-Plough & Doxorubicin & Pegylated liposome-im & $\begin{array}{l}\text { Metastatic breast and ovarian } \\
\text { cancer; Kaposi sarcoma }\end{array}$ & Marketed \\
\hline Myocet & ZeneusPharma Ltd & Doxorubicin & Liposoma/iv & Metastatic breast cancer & Marketed \\
\hline Doxil & Sequus Pharmaceutical & Doxorubicin & Liposome/iv & Kaposi sarcoma & Marketed \\
\hline L-Annamycin & Callisto Pharmaceuticals & Annamycin & Liposome/iv & $\begin{array}{l}\text { Children and young adults with } \\
\text { refractory or relapsed ALL or } A M L\end{array}$ & Phase I/II \\
\hline Genexol-PM & $\begin{array}{c}\text { Samyang } \\
\text { Pharmaceuticals }\end{array}$ & Paclitaxel & Methoxy PEG-PLA/iv & Breast and lung cancer & Phase II \\
\hline CALAA-01 & Calando Pharmaceuticals & $\begin{array}{l}\text { Anti-R2 } \\
\text { SiRNA }\end{array}$ & $\begin{array}{c}\text { Cyclodestrin-containing polymer (CAL } \\
\text { 101) and targeting agent (AD-PEG- } \\
\text { Tf)/iv }\end{array}$ & $\begin{array}{l}\text { Solid tumors that are refractory to } \\
\text { standard-of-care }\end{array}$ & Phase I \\
\hline Rexin-G & Epeius Biotechnologies & $\begin{array}{l}\text { Dominant negative cyclin } \\
\text { G1 construct }\end{array}$ & Pathotropic nanoparticles/iv & $\begin{array}{c}\text { Recurrent or metastatic breast } \\
\text { cancer }\end{array}$ & Phase I/II \\
\hline BikDD Nanoparticle & $\begin{array}{l}\text { MD Anderson Cancer } \\
\text { Center } / \mathrm{NCl}\end{array}$ & $\begin{array}{l}\text { Pro-apoptotic Bik gene } \\
\text { (BikDD) }\end{array}$ & Liposome/iv & Pancreatic Cancer & Phase I \\
\hline Docetaxel-PNP & Samyang & Docetaxel & Polymeric nanoparticles/iv & Advanced solid malignancies & Phase I \\
\hline
\end{tabular}

ROA: Route of administration, iv: intravenous, im: intramuscular, ALL: Acutelymphocyticleukemia, AML: Acutemyelogenousleukemia, PEG-PLA: Poly[ethylene glycol]poly[lactide], Tf: Human transferringprotein, HCC: Hepatocelular carcinoma 
nanoformulations currently available on the market is the table 1 [127].

Nanotechnology not only has the potential to conjugate the required targeting moiety, but also has the ability to carry the moiety for site-specific delivery without compromising its activity. Various polymeric materials are often used to synthesize nanoparticles loaded with conventional chemotherapy drugs such as docetaxel or doxorubicin, and then coated with polyethylene glycol to evade the patient's immune system. Additionally, nanoparticles can be conjugated with a targeted moiety such as an aptamer bioconjugated that binds, for example, to prostate-specific membrane antigens present on prostate cancer cells [128]. This type of active-targeted delivery to the tumor by using a tumor-specific moiety can be achieved by exploring various natural interactions like lectin-carbohydrate, ligand-receptor, and antibody-antigen interactions within the tumor cell [129], resulting in preferential accumulation within the cancerbearing organ or cancerous tumor cells. Active targeting has the potential to chance current cancer treatments scenarios.

Some nanoparticles have the ability to accumulate in tumor vasculature, known as enhanced permeability and retention (EPR) [130-132], thus increasing accumulation of the payload to the tumor site. Passive targeting in this case takes advantage of the rapid vascularization of hyper-permeable cells. This results in leaky, defective vessels and impaired lymphatic drainage. Nanoparticles sized at 10 to $100 \mathrm{~nm}$ have the ability to accumulate within tumors because of their ineffective lymphatic drainage. Thus, consideration of the size and surface properties of nanoparticles is vital, particularly for passive targeting. Particles must be less than $100 \mathrm{~nm}$ to avoid uptake by the reticulo-endothelial system and their surface should be hydrophilic to avoid rapid clearance by macrophages [133] Furthermore, both active and passive targeting can be exploited simultaneously to obtain maximum efficacy.

\section{Future perspective}

Nanotechnology has already made an impact on medicine. The rapid intrusion of this cutting-edge technology in the current stateof the-art has bring new avenues in the detection, treatment and therapy of cancer and due to this technologies are in preclinical stages there is still plenty of work to be done and some very promising new nanotechnology treatment methods are in the works. For instance, Singh et al. have recently developed Metal oxide nanoparticles (MONPs), which are multidisciplinary nano-scaled molecules used in the diagnosis and treatment of cancer. The MO-NPsin combination with other chemotherapeutic anticancer agents not only increase their bioavailability but also lower down the requirement of active dosages [134]. In this context, there is a tremendous potential for nanotechnology to enable cancer detection in its early stages. Most interesting is the potential to replace highly invasive conventional cancer detection and treatment, which often includes biopsies, irradiation, and painful therapies. As we become better able to engineer more sophisticated nanodevices and to equip them with effective targeting techniques for biomolecules, we will gain the ability to treat various kinds of cancer more and more effectively, there is no doubt that a bright future lies ahead for this discipline.

\section{Acknowledgements}

We thank INECOL, A. C. and CONACyT for the financial support.

\section{References}

1. Adeli M, Soleyman R, Beiranvand Z, Madani F (2013) Carbon nanotubes in cancer therapy: a more precise look at the role of carbon nanotube-polymer interactions. Chem Soc Rev 42: 5231-5256.

2. Bhushan B (2010) Introduction to Nanotechnology. In: Bhushan B, Springer Handbook of Nanotechnology. Springer Heidelberg Dordrecht London, New York, NY, 1964.
3. Vollath D (2013) Introduction to Nanoparticles and Nanomaterials. In: Horikoshi S, Serpone N, Microwaves in Nanoparticle Synthesis. ( $1^{\text {st }}$ edn), Wiley-VCH Verlag GmbH \& Co. KGaA, 1-24.

4. Chan HK, Kwok PC (2011) Production methods for nanodrug particles using the bottom-up approach. Adv Drug Deliv Rev 63: 406-416.

5. Brown SD, Nativo P, Smith JA, Stirling D, Edwards PR, et al. (2010) Gold nanoparticles for the improved anticancer drug delivery of the active component of oxaliplatin. J Am Chem Soc 132: 4678-4684.

6. Hoskins C (2014) The Use of Iron Oxide Nanoparticles for Pancreatic Cancer Therapy. J Nanomedicine Res 1: 1-17

7. Fan W, Shen B, Bu W, Zheng X, He Q, et al. (2015) Design of an intelligent sub-50 nm nuclear-targeting nanotheranostic system for imaging guided intranuclear radiosensitization. Chem Sci 6: 1747-1753.

8. De M, Ghosh PS, Rotello VM (2008) Applications of Nanoparticles in Biology. Adv Mater 20: 4225-4241.

9. Fukumori Y, Ichikawa H (2006) Nanoparticles for cancer therapy and diagnosis. Adv Powder Technol 17: 1-28.

10. Ficai D, Ficai A, Andronescu E (2015) Advances in Cancer Treatment: Role of Nanoparticles. In: Larramendy LM, Soloneski S, Nanomaterials - Toxicity and Risk Assessment. InTech, 1-22.

11. Huang G, Chen H, Dong Y, Luo X, Yu H, et al. (2013) Superparamagnetic iron oxide nanoparticles: amplifying ROS stress to improve anticancer drug efficacy. Theranostics 3: 116-126.

12. Liu L, Miao Q, Liang G (2013) Quantum Dots as Multifunctional Materials for Tumor Imaging and Therapy. Materials 6: 483-499.

13. Mangraviti A, Tzeng SY, Kozielski KL, Wang Y, Jin Y, et al. (2015) Polymeric nanoparticles for nonviral gene therapy extend brain tumor survival in vivo. ACS Nano 9: 1236-1249.

14. Ji SR, Liu C, Zhang B, Yang F, Xu J, et al. (2010) Carbon nanotubes in cancer diagnosis and therapy. Biochim Biophys Acta 1806: 29-35.

15. Andresen TL, Jensen SS, Jørgensen K (2005) Advanced strategies in liposomal cancer therapy: problems and prospects of active and tumor specific drug release. Prog Lipid Res 44: 68-97.

16. Merza KS, Al-Attabi HD, Abbas ZM, Yusr HA (2012) Comparative Study on Methods for Preparation of Gold Nanoparticles. Green Sustain Chem 2: 26-28.

17. Srivastava AK, Yadev R, Rai VN, Ganguly T, Deb SK (2012) Surface plasmon resonance in gold nanoparticles. AIP Conf Proceeding 1447: 305-306.

18. El-Brolossy TA, Abdallah T, Mohamed MB, Abdallah S, Easawi K, et al (2008) Shape and size dependence of the surface plasmon resonance of gold nanoparticles studied by Photoacoustic technique. Eur Phys J Spec Top 153: 361-364.

19. Khlebtsov NG, Dykman LA (2010) Optical properties and biomedical applications of plasmonic nanoparticles. J Quant Spectrosc Radiat Transf 111: 1-35.

20. Huang X, El-Sayed MA (2010) Gold nanoparticles: Optical properties and implementations in cancer diagnosis and photothermal therapy. J Adv Res 1: $13-28$.

21. Cherukuri P, Glazer ES, Curley SA (2010) Targeted hyperthermia using metal nanoparticles. Adv Drug Deliv Rev 62: 339-345.

22. Chithrani DB, Jelveh S, Jalali F, van Prooijen M, Allen C, et al. (2010) Gold nanoparticles as radiation sensitizers in cancer therapy. Radiat Res 173: 719728

23. Patra CR, Bhattacharya R, Mukhopadhyay D, Mukherjee P (2010) Fabrication of gold nanoparticles for targeted therapy in pancreatic cancer. Adv Drug Deliv Rev 62: 346-361

24. Kim D, Jon S (2012) Gold nanoparticles in image-guided cancer therapy. Inorganica Chim Acta 393: 154-164.

25. Figueroa ER, Lin AY, Yan J, Luo L, Foster AE, et al. (2014) Optimization of PAMAM-gold nanoparticle conjugation for gene therapy. Biomaterials 35 : 1725-1734.

26. Chanda N, Kan P, Watkinson LD, Shukla R, Zambre A, et al. (2010) Radioactive gold nanoparticles in cancer therapy: therapeutic efficacy studies of GA-198AuNP nanoconstruct in prostate tumor-bearing mice. Nanomedicine Nanotechnology Biol Med 6: 201-209.

27. Dickerson EB, Dreaden EC, Huang X, El-Sayed IH, Chu H, et al. (2008) Gold nanorod assisted near-infrared plasmonic photothermal therapy (PPTT) of squamous cell carcinoma in mice. Cancer Lett 269: 57-66.

28. Huang X, El-Sayed MA (2011) Plasmonic photo-thermal therapy (PPTT) Alexandria J Med 47: 1-9. 
29. Mackey MA, Ali MR, Austin LA, Near RD, El-Sayed MA (2014) The most effective gold nanorod size for plasmonic photothermal therapy: theory and in vitro experiments. J Phys Chem B 118: 1319-1326.

30. Guo H, Barnard AS (2013) Naturally occurring iron oxide nanoparticles: morphology, surface chemistry and environmental stability. J Mater Chem A 1: $27-42$.

31. Huber DL (2005) Synthesis, properties, and applications of iron nanoparticles Small 1: 482-501.

32. Mohapatra M, Anand S (2010) Synthesis and applications of nano-structured iron oxides / hydroxides - a review. Int J Eng Sci Technol 2: 127-146.

33. Gupta AK, Gupta M (2005) Synthesis and surface engineering of iron oxide nanoparticles for biomedical applications. Biomaterials 26: 3995-4021.

34. Lodhia J, Mandarano G, Ferris Nj, Eu P, Cowell S (2010) Development and use of iron oxide nanoparticles (Part 1): Synthesis of iron oxide nanoparticles for MRI. Biomed Imaging Interv J 6: e12.

35. Yang C, Wu J, Hou Y (2011) Fe3O4 nanostructures: synthesis, growth mechanism, properties and applications. Chem Commun (Camb) 47: 5130 5141.

36. Lee H, Yu MK, Park S, Moon S, Min JJ, et al. (2007) Thermally cross-linked superparamagnetic iron oxide nanoparticles: synthesis and application as a dual imaging probe for cancer in vivo. J Am Chem Soc 129: 12739-12745.

37. Laurent S, Dutz S, Häfeli UO, Mahmoudi M (2011) Magnetic fluid hyperthermia: focus on superparamagnetic iron oxide nanoparticles. Adv Colloid Interface Sci 166: 8-23.

38. Yang HW, Hua MY, Liu HL, Huang CY, Wei KC (2012) Potential of magnetic nanoparticles for targeted drug delivery. Nanotechnol Sci Appl 5: 73-86.

39. Mahmoudi M, Sant S, Wang B, Laurent S, Sen T (2011) Superparamagnetic iron oxide nanoparticles (SPIONs): development, surface modification and applications in chemotherapy. Adv Drug Deliv Rev 63: 24-46.

40. Zhang S, Zhao X, Niu H, Shi Y, Cai Y, et al. (2009) Superparamagnetic $\mathrm{Fe} 3 \mathrm{O} 4$ nanoparticles as catalysts for the catalytic oxidation of phenolic and aniline compounds. J Hazard Mater 167: 560-566.

41. Peng FF, Zhang Y, Gu N (2008) Size-dependent peroxidase-like catalytic activity of Fe3O4 nanoparticles. Chinese Chem Lett 19: 730-733.

42. Bera D, Qian L, Tseng TK, Holloway PH (2010) Quantum dots and their multimodal applications: A review. Materials 3: 2260-2345.

43. Lemon CM, Curtin PN, Somers RC, Greytak AB, Lanning RM, et al. (2014) Metabolic tumor profiling with $\mathrm{pH}$, oxygen, and glucose chemosensors on quantum dot scaffold. Inorg Chem 53: 1900-1915.

44. Hines DA, Kamat PV (2014) Recent advances in quantum dot surface chemistry. ACS Appl Mater Interfaces 6: 3041-3057.

45. Gerion D, Pinaud F, Williams SC, Parak WJ, Zanchet D, et al. (2001) Synthesis and Properties of Biocompatible Water-Soluble Silica-Coated CdSe / ZnS Semiconductor Quantum Dots. J Phys Chem B 105: 8861-8871.

46. Nordell KJ, Boatman EM, Lisensky GC (2005) A Safer, Easier, Faster Synthesis for CdSe Quantum Dot Nanocrystals. J Chem Educ 82: 1697.

47. Chang YP, Pinaud F, Antelman J, Weiss S (2008) Tracking bio-molecules in live cells using quantum dots. J Biophotonics 1: 287-298.

48. Barroso MM (2011) Quantum dots in cell biology. J Histochem Cytochem 59: $237-251$

49. Schroeder JE, Shweky I, Shmeeda H, Banin U, Gabizon A (2007) Folatemediated tumor cell uptake of quantum dots entrapped in lipid nanoparticles. J Control Release 124: 28-34.

50. Erogbogbo F, Yong KT, Roy I, Xu G, Prasad PN, et al. (2008) Biocompatible luminescent silicon quantum dots for imaging of cancer cells. ACS Nano 2 873-878.

51. Mikhaylova M, Kim DK, Bobrysheva N, Osmolowsky M, Semenov V, et al. (2004) Superparamagnetism of magnetite nanoparticles: dependence on surface modification. Langmuir 20: 2472-2477.

52. Tan WB, Zhang $Y$ (2005) Surface modification of gold and quantum dot nanoparticles with chitosan for bioapplications. J Biomed Mater Res A 75 : 56-62.

53. Duncan R (2003) The dawning era of polymer therapeutics. Nat Rev Drug Discov 2: 347-360.

54. Torchilin VP (2002) PEG-based micelles as carriers of contrast agents for different imaging modalities. Adv Drug Deliv Rev 54: 235-252.

55. Lee ES, Na K, Bae YH (2005) Super pH-sensitive multifunctional polymeric micelle. Nano Lett 5: 325-329.
56. Gaucher G, Dufresne MH, Sant VP, Kang N, Maysinger D, et al. (2005) Block copolymer micelles: preparation, characterization and application in drug delivery. J Control Release 109: 169-188.

57. Couvreur P, Dubernet C, Puisieux F (1995) Controlled drug delivery with nanoparticles?: current possibilities and future trends. Eur J Pharm Biopharm 41: 2-13.

58. Jung T, Kamm W, Breitenbach A, Kaiserling E, Xiao JX, et al. (2000) Biodegradable nanoparticles for oral delivery of peptides: is there a role for polymers to affect mucosal uptake? Eur J Pharm Biopharm 50: 147-160.

59. Kreuter J (1990) Large-scale production problems and manufacturing of nanoparticles. In: Marcel Dekker, Specialized drug delivery system. New York, 257.

60. Kreuter J (1991) Nanoparticle-based drug delivery systems. J Control Release 16: 169-176.

61. Vauthier C, Dubernet C, Fattal E, Pinto-Alphandary H, Couvreur P (2003) Poly(alkylcyanoacrylates) as biodegradable materials for biomedical applications. Adv Drug Deliv Rev 55: 519-548.

62. Tice TR, Gilley RM (1985) Preparation of injectable controlled-release microcapsules by a solvent-evaporation process. J Control Release 2: 343-352.

63. Soppimath KS, Aminabhavi TM, Kulkarni AR, Rudzinski WE (2001) Biodegradable polymeric nanoparticles as drug delivery devices. J Control Release 70: 1-20.

64. Quintanar-Guerrero D, Allémann E, Fessi H, Doelker E (1998) Preparation techniques and mechanisms of formation of biodegradable nanoparticles from preformed polymers. Drug Dev Ind Pharm 24: 1113-1128.

65. Barichello JM, Morishita M, Takayama K, Nagai T (1999) Encapsulation of hydrophilic and lipophilic drugs in PLGA nanoparticles by the nanoprecipitation method. Drug Dev Ind Pharm 25: 471-476.

66. You J, Li W, Yu C, Zhao C, Jin L, et al. (2013) Amphiphilically modified chitosan cationic nanoparticles for drug delivery. J Nanoparticle Res 15: 2123.

67. Huang S, Wan Y, Wang Z, Wu J (2013) Folate-conjugated chitosanpolylactide nanoparticles for enhanced intracellular uptake of anticancer drug. J Nanoparticle Res 15: 2096.

68. Park JH, Cho YW, Son YJ, Kim K, Chung H, et al. (2006) Preparation and characterization of self-assembled nanoparticles based on glycol chitosan bearing adriamycin. Colloid Polym Sci 284: 763-770.

69. Langner M, Kral TE (1999) Liposome-based drug delivery systems. Pol J Pharmacol 51: 211-222.

70. Seltzer SE (1988) Contrast-carrying liposomes. Current status. Invest Radio 23 Suppl 1: S122-125.

71. Seltzer SE, Swensson RG, Judy PF, Nawfel RD (1988) Size discrimination in computed tomographic images. Effects of feature contrast and display window. Invest Radiol 23: 455-462.

72. Adzamli IK, Seltzer SE, Slifkin M, Blau M, Adams DF (1990) Production and characterization of improved liposomes containing radiographic contrast media. Invest Radiol 25: 1217-1223.

73. Simon RH, Ho SY, D'Arrigo J, Wakefield A, Hamilton SG (1990) Lipid-coated ultrastable microbubbles as a contrast agent in neurosonography. Invest Radiol 25: 1300-1304

74. Uziely B, Jeffers S, Isacson R, Kutsch K, Wei-Tsao D, et al. (1995) Liposomal doxorubicin: antitumor activity and unique toxicities during two complementary phase I studies. J Clin Oncol 13: 1777-1785.

75. Northfelt DW, Dezube BJ, Thommes JA, Levine R, Von Roenn JH, et al (1997) Efficacy of pegylated-liposomal doxorubicin in the treatment of AIDSrelated Kaposi's sarcoma after failure of standard chemotherapy. J Clin Oncol 15: 653-659.

76. Ranson MR, Carmichael J, O'Byrne K, Stewart S, Smith D, et al. (1997) Treatment of advanced breast cancer with sterically stabilized liposomal doxorubicin: results of a multicenter phase II trial. J Clin Oncol 15: 3185-3191.

77. Muggia FM, Hainsworth JD, Jeffers S, Miller P, Groshen S, et al. (1997) Phase II study of liposomal doxorubicin in refractory ovarian cancer: antitumor activity and toxicity modification by liposomal encapsulation. J Clin Oncol 15: 987-993.

78. Bulmus V, Woodward M, Lin L, Murthy N, Stayton P, et al. (2003) A new $\mathrm{pH}$-responsive and glutathione-reactive, endosomal membrane-disruptive polymeric carrier for intracellular delivery of biomolecular drugs. J Control Release 93: 105-120.

79. Bae Y, Jang WD, Nishiyama N, Fukushima S, Kataoka K (2005) Multifunctional polymeric micelles with folate-mediated cancer cell targeting and $\mathrm{pH}$-triggered drug releasing properties for active intracellular drug delivery. Mol Biosyst 1: 242-250. 
80. Allen C, Han J, Yu Y, Maysinger D, Eisenberg A (2000) Polycaprolactoneb-poly(ethylene oxide) copolymer micelles as a delivery vehicle for dihydrotestosterone. J Control Release 63: 275-286.

81. La SB, Okano T, Kataoka K (1996) Preparation and characterization of the micelle-forming polymeric drug indomethacin-incorporated poly(ethylene oxide)-poly(beta-benzyl L-aspartate) block copolymer micelles. J Pharm Sc 85: $85-90$

82. Wang F, Bronich TK, Kabanov AV, Rauh RD, Roovers J (2005) Synthesis and evaluation of a star amphiphilic block copolymer from poly(epsiloncaprolactone) and poly(ethylene glycol) as a potential drug delivery carrier Bioconjug Chem 16: 397-405.

83. Lavasanifar A, Samuel J, Kwon GS (2001) Micelles self-assembled from poly(ethylene oxide)-block-poly( $\mathrm{N}$-hexyl stearate $\mathrm{L}$-aspartamide) by a solven evaporation method: effect on the solubilization and haemolytic activity of amphotericin B. J Control Release 77: 155-160.

84. Lee ES, Na K, Bae YH (2005) Doxorubicin loaded pH-sensitive polymeric micelles for reversal of resistant MCF-7 tumor. J Control Release 103: 405 418

85. Yoo HS, Park TG (2004) Folate receptor targeted biodegradable polymeric doxorubicin micelles. J Control Release 96: 273-283

86. Yokoyama M, Okano T, Sakurai Y, Suwa S, Kataoka K (1996) Introduction of cisplatin into polymeric micelle. J Control Release 39: 351-356.

87. Gusberg S, CD Runowicz (1991) Gynecologic cancers. In: Holleb AI, Fink DJ Murphy GP American Cancer Society textbook of clinical oncology. American Cancer Society, Atlanta, 482

88. Sherman SE, Lippard SJ (1987) Structural aspects of platinum anticancer drug interactions with DNA. Chem Rev 87: 1153-1181.

89. Nishiyama N, Kataoka K (2001) Preparation and characterization of sizecontrolled polymeric micelle containing cis-dichlorodiammineplatinum(II) in the core. J Control Release 74: 83-94.

90. Nishiyama N, Okazaki S, Cabral H, Miyamoto M, Kato Y, et al. (2003) Novel cisplatin-incorporated polymeric micelles can eradicate solid tumors in mice. Cancer Res 63: 8977-8983.

91. Triesscheijn M, Baas P, Schellens JH, Stewart FA (2006) Photodynamic therapy in oncology. Oncologist 11: 1034-1044.

92. Roy I, Ohulchanskyy TY, Pudavar HE, Bergey EJ, Oseroff AR, et al. (2003) Ceramic-Based Nanoparticles Entrapping Water-Insoluble Photosensitizing Anticancer Drugs: A Novel Drug-Carrier System for Photodynamic Therapy. J Am Chem Soc 125: 7860-7865.

93. Henderson BW, Bellnier DA, Greco WR, Sharma A, Pandey RK, et al. (1997) An in vivo quantitative structure-activity relationship for a congeneric series of pyropheophorbide derivatives as photosensitizers for photodynamic therapy. Cancer Res 57: 4000-4007

94. Ohulchanskyy TY, Roy I, Goswami LN, Chen Y, Bergey EJ, et al. (2007) Organically modified silica nanoparticles with covalently incorporated photosensitizer for photodynamic therapy of cancer. Nano Lett 7: 2835-2842.

95. Bechet D, Couleaud P, Frochot C, Viriot ML, Guillemin F, et al. (2008) Nanoparticles as vehicles for delivery of photodynamic therapy agents. Trends Biotechnol 26: 612-621.

96. Ajaykumar B, Babu NVN, Reddi L, Ujwala K (2012) Applications of dendrimers in cancer therapy. Int. J Chem Pharm Sci 3: 1-7.

97. Gardikis K, Hatziantoniou S, Bucos M, Fessas D, Signorelli M, et al. (2010) New drug delivery nanosystem combining liposomal and dendrimeric technology (liposomal locked-in dendrimers) for cancer therapy. J Pharm Sci 99: 3561-3571.

98. Kuo JH, Liou MJ, Chiu HC (2010) Evaluating the gene-expression profiles of HeLa cancer cells treated with activated and nonactivated poly(amidoamine) dendrimers, and their DNA complexes. Mol Pharm 7: 805-814.

99. Bardhan R, Chen W, Perez-Torres C, Bartels M, Huschka RM, et al. (2009) Nanoshells with Targeted Simultaneous Enhancement of Magnetic and Optical Imaging and Photothermal Therapeutic Response. Adv Funct Mater 19: 3901-3909

100. Choi MR, Stanton-Maxey KJ, Stanley JK, Levin CS, Bardhan R, et al. (2007) A cellular Trojan Horse for delivery of therapeutic nanoparticles into tumors. Nano Lett 7: 3759-3765.

101.Bardhan R, Grady NK, Halas NJ (2008) Nanoscale control of near-infrared fluorescence enhancement using Au nanoshells. Small 4: 1716-1722.

102. Ma M, Chen H, Chen Y, Wang X, Chen F, et al. (2012) Au capped magnetic core/mesoporous silica shell nanoparticles for combined photothermo-/ chemo-therapy and multimodal imaging. Biomaterials 33: 989-998.
103. Thakare VS, Das M, Jain AK, Patil S, Jain S (2010) Carbon nanotubes in cancer theragnosis. Nanomedicine (Lond) 5: 1277-1301.

104. Chen J, Chen S, Zhao X, Kuznetsova LV, Wong SS, et al. (2008) Functionalized single-walled carbon nanotubes as rationally designed vehicles for tumor-targeted drug delivery. J Am Chem Soc 130: 1677816785.

105.Li S, He P, Dong J, Guo Z, Dai L (2005) DNA-directed self-assembling of carbon nanotubes. J Am Chem Soc 127: 14-15.

106. Wang X, Liu F, Andavan GT, Jing X, Singh K, et al. (2006) Carbon nanotubeDNA nanoarchitectures and electronic functionality. Small 2: 1356-1365.

107. Han X, Li Y, Deng Z (2007) DNA-Wrapped Single Walled Carbon Nanotubes as Rigid Templates for Assembling Linear Gold Nanoparticle Arrays. Adv Mater 19: 1518-1522.

108. Gao H, Kong Y, Cui D, Ozkan CS (2003) Spontaneous Insertion of DNA Oligonucleotides into Carbon Nanotubes. Nano Lett 3: 471-473.

109. Kam NW, O'Connell M, Wisdom JA, Dai H (2005) Carbon nanotubes as multifunctional biological transporters and near-infrared agents for selective cancer cell destruction. Proc Natl Acad Sci U S A 102: 11600-11605.

110. Gao X, Cui Y, Levenson RM, Chung LWK, Nie S (2004) In vivo cancer targeting and imaging with semiconductor quantum dots. Nat Biotechnol 22: 969-976.

111. Jain TK, Morales MA, Sahoo SK, Leslie-Pelecky DL, Labhasetwar V (2005) Iron oxide nanoparticles for sustained delivery of anti- cancer agents. Mol Pharm 2: 194-205.

112. Pelicano H, Martin DS, Xu RH, Huang P (2006) Glycolysis inhibition for anticancer treatment. Oncogene 25: 4633-4646.

113. Deryugina EI, Quigley JP (2006) Matrix metallo-proteinases and tumor metastasis. Cancer Metastasis Rev 25: 9-34.

114. Edens HA, Levi BP, Jaye DL, Walsh S, Reaves TA, et al. (2002) Neutrophil transepithelial migration: evidence for sequential, contact-dependent signaling events and enhanced paracellular permeability independent of transjunc- tional migration. J Immunol 169: 476-486.

115. Jain RK (1987) Transport of molecules across tumor vasculature. Cancer Metastasis Rev 6: 559-593.

116. Northfelt DW, Martin FJ, Working P, Volberding PA, Russell J, et al. (1996) Doxorubicin encapsulated in liposomes contain- ing surface-bound polyethylene glycol: pharma- cokinetics, tumor localization, and safety in patients with AIDS-related Kaposi's sarcoma. J Clin Pharmacol 36: 55-63.

117. Vicent MJ, Duncan R (2006) Polymer conjugates: nanosized medicines for treating cancer. Trends Biotechnol 24: 39-47.

118. Shapira A, Livney YD, Broxterman HJ, Assaraf YG (2011) Nanomedicine for targeted cancer therapy: towards the overcoming of drug resistance. Drug Resist Updat 14: 150-163.

119. Wittamer V, Bertrand JY, Gutschow PW, Traver D (2011) Characterization of the mononuclear phagocyte system in zebrafish. Blood 117: 7126-7135.

120. Owens DE $3^{\text {rd }}$, Peppas NA (2006) Opsonization, biodistribution, and pharmacokinetics of polymeric nanoparticles. Int J Pharm 307: 93-102.

121. Yagoda A, Petrylak D (1993) Cytotoxic chemotherapy for advanced hormone-resistant prostate cancer. Cancer 71: 1098-1109.

122. Li SD, Huang L (2008) Pharmacokinetics and biodistribution of nanoparticles Mol Pharm 5: 496-504.

123. Nishiyama N, Kataoka K (2006) Current state, achievements, and future prospects of polymeric micelles as nanocarriers for drug and gene delivery. Pharmacol Ther 112: 630-648.

124. Sparreboom A, Scripture CD, Trieu V, Williams PJ, De T, et al. (2005) Comparative preclinical and clinical pharmacokinetics of a cremophor-free, nanoparticle albumin-bound paclitaxel $(\mathrm{ABI}-007)$ and paclitaxel formulated in Cremophor (Taxol). Clin Cancer Res 11: 4136-4143.

125. Gradishar WJ, Tjulandin S, Davidson N, Shaw H, Desai N, et al. (2005) Phase III trial of nanoparticle albumin-bound paclitaxel compared with polyethylated castor oil-based paclitaxel in women with breast cancer. $\mathrm{J}$ Clin Oncol 23: 7794-7803.

126. Moreno-Aspitia A Perez EA (2005) Nanoparticle albumin-bound paclitaxel (ABI007): a newer taxane alternative in breast cancer. Future Oncol 1: 755-762.

127. Bharali DJ, Mousa SA (2010) Emerging nanomedicines for early cancer detection and improved treatment: current perspective and future promise. Pharmacol Ther 128: 324-335.

128. Farokhzad OC, Cheng J, Teply BA, Sherifi I, Jon S, et al. (2006) Targeted nanoparticle-aptamer bioconjugates for cancer chemotherapy in vivo. Proc Natl Acad Sci USA 103: 6315-6320. 
129. Guo X, Szoka FC Jr (2003) Chemical approaches to triggerable lipid vesicles for drug and gene delivery. Acc Chem Res 36: 335-341.

130. Iyer AK, Khaled G, Fang J, Maeda H (2006) Exploiting the enhanced permeability and retention effect for tumor targeting. Drug Discov Today 11 812-818.

131. Maeda H, Wu J, Sawa T, Matsumura Y, Hori K (2000) Tumor vascular permeability and the EPR effect in macromolecular therapeutics: a review. J Contr Rel 65: 271-284.
132. Nam HY, Kwon SM, Chung H, Lee SY, Kwon SH, et al. (2009) Cellular uptake mechanism and intracellular fate of hydrophobically modified glycol chitosan nanoparticles. J Contr Rel 135: 259-267.

133. Jain RK (1999) Transport of molecules, particles, and cells in solid tumors Annu Rev Biomed Eng 1: 241-263.

134. Singh TH, Dharambir K, Kaur BS, Pardeep K, Gaurav K, et al. (2015) Molecular aspects of metal oxide nanoparticles (MO-Nps) mediated pharmacological effects. Life sciences 143: 71-79. 